\title{
Artificial Intelligence Suggests that UAE Needs to Mitigate the Small COVID-19 Resurgence
}

\author{
Yoshiyasu Takefuji \\ Faculty of Environment and Information Studies, Keio University, Fujisawa, Japan
}

Since we have no vaccine and no effective therapy against the COVID-19, we have one and only one policy based on isolating-all-at-risk strategy [1]. The purpose of this manuscript is to apply the best digital fence to UAE. The success of a digital fence in Taiwan was brought by bold political decisions for border control, case identification, containment, and resource allocation, respectively [2]. The basis for such political decisions was technical aspects such as massive testing results, mathematical models of infectious diseases, and information sharing using digital technology [2]. Isolation surveillance is called digital fence where smartphones can be used for providing the location of suspected patients or patients in real time. As much as possible, we should maximize the digital surveillance coverage. The best isolation surveillance using coronavirus Apps is implemented by Taiwan with more than $99 \%$ of coverage which is a key to the success [3]. Taiwan is tracking 55,000 people under a mandatory 14-day home quarantine in real time [3]. The consequence of Taiwan policy shows only 7 deaths due to the COVID-19 as of December 29 in 2020 with a population of 23.8 million [4]. UAE does not use mandatory coronavirus Apps [1]. In other words, the UAE policy is based on voluntary coronavirus Apps so that digital fence of UAE is leaky.

\section{karger@karger.com www.karger.com/dmj

Many nations implement the similar digital isolation surveillance but their coverage is much smaller than that of Taiwan. UAE with a population of 9.95 million has 662 deaths due to the COVID-19 as of December 30 in 2020 [4]. UAE is composed of 7 independent city-states so that sharing the isolation surveillance data between them plays a key role in mitigating the pandemic.

In this manuscript, the state-of-the-art artificial intelligence algorithm is used for predicting the number of deaths in the near future. The algorithm is based on curve fitting with a variety of degree polynomials. In regression analysis, curve fitting is the process of specifying the model that provides the best fit to the specific curves in a given dataset. Figure 1 shows that the small resurgence can be observed in the number of daily deaths in UAE where $X$-axis indicates the xth day from January 22 in 2020 and $Y$-axis the number of daily deaths due to the COVID- 19 . In Figure 1, black line indicates the number of daily deaths, while blue line based on the 10th degree polynomial shows the predicted result using a dataset of the last 265 days from December 28 in 2020 for curve-fitting machine learning, redline based on the 5 th degree polynomial changing the dataset size from 120 to 265 days from December 28 in 2020. The new_deaths.csv file data was downloaded [4] and used for curve-fitting machine learn-

Correspondence to:

Yoshiyasu Takefuji, takefuji@keio.jp 
Fig. 1. The number of daily deaths due to COVID-19 in UAE as of January 26, 2021. In the graph, black line: the number of daily deaths, blue line: predicted curve using 300 days based on 10th degree polynomial curve fitting, redline changing the number of days from 100 to 300 days from January 26,2021 , based on 5 th degree polynomial curve fitting (gif animation file is at: https:// github.com/ytakefuji/uae/blob/main/uae. gif).

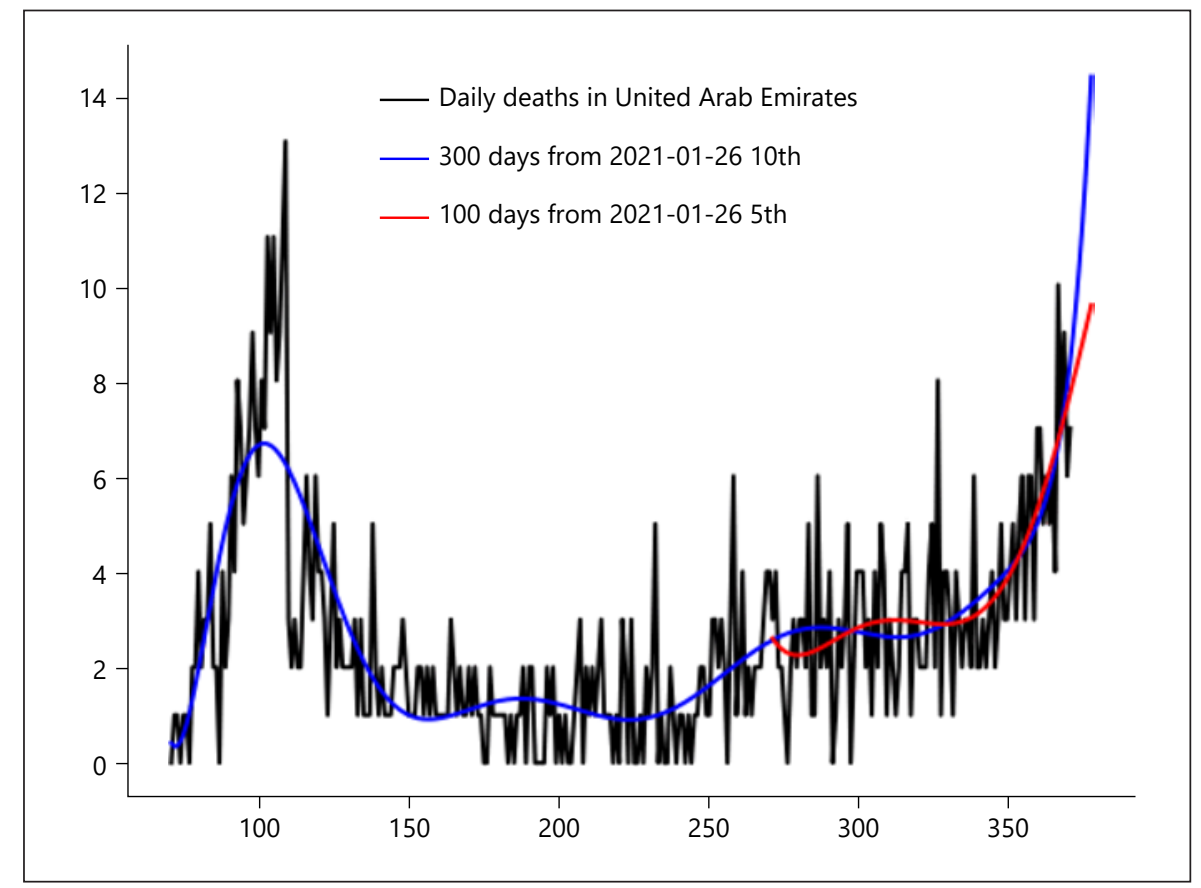

ing. The curve-fitting program, animedaily.py is available at the github site [5].

Based on the result, the data size and the degree size of polynomial can produce a variety of predictions. The less data, the recent trends will be emphasized in the predicted graph as shown in Figure 1. Policymakers can observe a variety of predicted behaviors in order to control the pandemic whether they should strengthen their policy or not.

\section{Discussion}

The starting point of the technical side of digital fence in Taiwan is based on massive testing. The essence of digital fence lies in grasping the whole features of the pandemic using massive testing, which becomes the input value of the mathematical model of infectious disease and to share it digitally and use it as a material for policy judgment. Although the very poor detection accuracy of PCR is an undeniable fact [6], the effectiveness of PCR testing with massive testing has been pointed out [7-10].

Kuniya and Inaba examined the effectiveness of suppressing the epidemic of massive testing by using a mathematical model to examine the effectiveness of isolation policies through routine testing and confirmed the effectiveness of massive testing regardless of the epidemic scale [11].

Small Resurgence in UAE

\section{Rationale}

The rationale for the proposed mathematical model of infectious diseases is addressed. There was no way to describe the dynamics of infection during the Spanish influenza epidemic 100 years ago, in terms of controlling a nonchemical outbreak during the early stages of the epidemic, when vaccines were not developed. Then, the SIR model was introduced with a differential equation describing a single outbreak in an artificial [12]. The differential equations have been extensively improved by Diekmann et al. [13]. There is an example of monitoring using the differential equation system like Hamzah et al. [14] in COVID-19 at present time.

However, there is criticism that if the infectious disease estimation by the differential equation method is performed with fixed parameters, the infection of a certain population is calculated before the natural convergence of the infection. In other words, in infectious diseases where the mortality rate cannot be ignored, people do not take infection-avoidance actions, and the transmission coefficient, which includes the social variable of contact frequency, changes over time and cannot be a fixed parameter. Host populations are not uniformly mixed, and the size of the population involved in the infection process itself may vary with policy intervention effects and avoidance behavior. 
On the other hand, some mathematical models of infectious diseases take a statistical approach to estimate quantitative profiles from infection status data, as adopted by the proposed study. Although this mathematical statistical method is not necessarily able to understand the phenomena of infectious diseases like the differential equation method, its evaluation has been established as a methodology which sufficiently supplements the criticism toward the differential equation method. It should be emphasized in advance that understanding of the phenomena of infectious diseases is not underestimated, but for monitoring constituting digital fence, it is possible to express a realistic infection situation by a mathematical statistical method. method. This study not only discussed the problems and the direction of the solution on the technical side as a key of the introduction of Digital fence but also showed the concrete result on the infectious disease mathematical model. In this research, this paper presented bold political judgments for border control, case identification, containment, and resource allocation, which constitute the digital fence, and the supporting technical aspects such as massive testing, a mathematical model of infectious diseases, and information sharing by digital technology.

\section{Conflict of Interest Statement}

The author has no conflicts of interest to declare.

\section{Funding Sources}

The author did not receive any funding. tial equation method and the mathematical statistical

This manuscript clarifies that there are two types of mathematical models for infectious diseases, the differen-

\section{References}

1 Laney Zhang, Taiwan. Regulating Electronic Means to Fight the Spread of COVID-19. https: //www.loc.gov/law/help/coronavirusapps/taiwan.php https://www.loc.gov/law/ help/coronavirus-apps/coronavirus-apps. pdf.

2 Wang J, Ng CY. RH brook response to COVID-19 in Taiwan: big data analytics, new technology, and proactive testing. JAMA. 2020;323:1341-2.

3 Remarks by Dr. Jaushieh Joseph Wu, Minister of Foreign Affairs, R.O.C (Taiwan) for the "Taiwan's Strong COVID-19 Responses" online symposium hosted by the Hudson Institute April 9, 2020. https://www.mofa.gov.tw/ Upload/RelFile/662/171747/61019a02-. 51c9--4e73--9015-d50256b3fef0.pdf.

4 JHU. Daily deaths of COVID-19. https://raw. githubusercontent.com/owid/covid-19-data/ master/public/data/jhu/new_deaths.csv (Accessed 2020 December 30).
5 Takefuji Y., UAE COVID-19 prediction. https://github.com/ytakefuji/uae.

6 Long C, Xu H, Shen Q, Zhang X, Fan B, Wang $\mathrm{C}$, et al. Diagnosis of the Coronavirus disease (COVID-19): rRT-PCR or CT? Eur J Radiol. 2020;126:108961.

7 Allen D, et al. National Covid-19 testing action plan pragmatic steps to reopen our workplaces and our communities. New York: Rockefeller Foundation; 2020.

8 Allen D, et al. Roadmap to pandemic resilience, safra center for ethics. Cambridge: Harvard University; 2020.

9 Peto J, et al. Stopping the lockdown and ending the epidemic by universal weekly testing as the exit strategy. 2020. https://ephg-covid-19.org/.

10 Romer P. Roadmap to responsibly reopen America. 2020. https://roadmap.paulromer. net/.

11 Kuniya T, Inaba H. Possible effects of mixed prevention strategy for COVID-19 epidemic: massive testing, quarantine and so-cial distancing. AIMS Public Health. 2020;7:490503.

12 Kermack WO, McKendrick AG. Contributions to the mathematical theory of epidemics I. Proc R Soc London Ser A. 1927;115A:70021. reprinted in Bull Math Biol. 1991;53(1/2): 33-55.

13 Diekmann O, Heesterbeek JA, Metz JAJ. On the definition and the computation of the basic reproduction ratio R0 in models for infectious diseases in heterogeneous populations. J Math Biol. 1990;28(4):365-82.

14 Binti Hamzah FA, Lau C, Nazri H, Ligot DV, Lee G, Tan CL, et al. CoronaTracker: worldwide COVID-19 outbreak data analysis and prediction. [Preprint]. Bull World Health Organ. 2020 Mar 19. Epub. 